\title{
Recurrent intrathecal catheter-tip granuloma in a patient receiving high dose hydromorphone: A case report
}

\author{
Teena Varghese $^{1}$, Joshua Bemporad ${ }^{2}$, Stefano Camici ${ }^{2}$, Steven Mortazavi ${ }^{1}$ \\ ${ }^{1}$ Valley Pain Specialists PC, Bethlehem, USA \\ ${ }^{2}$ Lehigh Valley Hospital and Health Network, Allentown, USA \\ Email: steven_mortazavi@valleypainspecialists.com
}

Received 15 November 2012; revised 24 December 2012; accepted 11 January 2013

\begin{abstract}
Study Design: Case report. Patient Sample: A 42year-old Caucasian male. Results: Catheter-tip granuloma formation is possible despite a normal catheter access study in patients with intrathecal (IT) infusion systems and its recurrence is possible after surgical excision. Increasing concentrations of hydromorphone from $50 \mathrm{mg} / \mathrm{ml}$ to $100 \mathrm{mg} / \mathrm{ml}$ without altering the daily dosage may have precipitated granuloma formation. Conclusions: In patients with previously implanted spinal catheters for intrathecal drug delivery, catheter access studies cannot be relied upon to rule out catheter-tip granulomas, which should be included in the differential diagnosis in patients with worsening clinical conditions or new onset neurologic symptoms. Caution should be exercised when increasing concentrations of intrathecal opioids while monitoring for signs or symptoms of intrathecal cathetertip granuloma formation. Removal of previously implanted catheters and/or reductions in opioid concentrations may be necessary to prevent recurrent granuloma formations, which can occur quickly after surgical excision.
\end{abstract}

Keywords: Catheter-Tip Granuloma; Intrathecal; Hydromorphone; Morphine; Spinal Cord; Catheter Access Study

\section{INTRODUCTION}

Intrathecal drug infusion systems have been utilized since the 1980 s to manage a variety of chronic pain conditions and spasticity. Thus far, over 150,000 pumps have been implanted worldwide [1]. An intrathecal (IT) granuloma is a well described condition which can result in serious, irreversible neurological deficits in patients with implanted subarachnoid catheters for opioid infusion therapy. The development of an inflammatory

\footnotetext{
${ }^{*}$ The author(s) received no financial support and have no conflict of
} interest to disclose. mass at the tip of an intrathecal catheter in patients receiving high dose narcotics was first described in 1991 [2]. It has been published less frequently in radiology journals [3] and is still being mistaken for other processes such as a hematoma on magnetic resonance imaging (MRI).

Although often associated with high concentrations of opioid therapy, most commonly morphine, other medications such as hydromorphone may result in catheter granulomas near the tip of the subarachnoid catheter $[1,4]$. For example, according to a case review of 41 patients, 31 patients receiving morphine and 9 patients receiving hydromorphone intrathecally were found to have inflammatory mass lesions at the catheter tip [5]. Another likely reason for the majority of patients receiving morphine is that it is the only opioid approved by the Food and Drug Administration for delivery of continuous drugs intrathecally [4].

\section{CASE REPORT}

In the current case, we report a 42-year-old male who presented to our pain clinic in 2009 with chronic low back pain, which began after he was rear-ended in a motor vehicle accident in 1993 while at work. He eventually went on to have a lumbar laminectomy in 1994 followed by an L4-5, L5-S1 fusion in 1995. However, due to ongoing chronic low back pain, the patient then went on to have a spinal cord stimulator implanted which was later removed within one year due to lack of efficacy. Subsequently an intrathecal pump and subarachnoid catheter was placed in August 1997 by an another pain management physician which provided good relief of pain. His pump was replaced in 2001 for normal end of life battery and pump site revision one year later due to pump discomfort after losing over 100 lbs of weight due to respiratory illness and development of skin excoriation over the pump site. The patient also underwent catheter removal and replacement at that time with the catheter tip placed to approximately $\mathrm{T} 5$ spinal level. However, the patient still continued to have dif- 
ficulty ambulating and decreased sensation in his lower extremities, thus leading him to utilize a wheel-chair for next 7 years.

At the initial visit in our clinic in 2009, the patient complained of 10/10 low back pain, right greater than left, radiating down bilateral lower extremities described as sharp, stabbing, burning, aching and constant. In addition, the patient reported weakness and numbness in his bilateral lower extremities, but no bowel or bladder incontinence. On physical exam, the patient had mild tenderness of bilateral iliolumbar ligaments, sciatic notch and SI joint as well as mild limitation of flexion, extension and bilateral lateral flexion with pain. In addition, the patient had a positive straight leg raise bilaterally as well as hyperesthesia on the left L4, L5 and S1 and motor strength of $4+/ 5$ for the bilateral lower extremities. Reflexes were intact with negative clonus and Babinski.

Examination of available pump telemetry printouts indicate that the patient was receiving intrathecal hydromorphone $50 \mathrm{mg} / \mathrm{ml}$ at a daily rate of $19.00 \mathrm{mg} /$ day as early as March 2000, prior to revision of his original pump. Prior to this, the patient was receiving intrathecal morphine $50 \mathrm{mg} / \mathrm{ml}$ with clonidine $30 \mathrm{mcg} / \mathrm{ml}$ at a dose of $32 \mathrm{mg} /$ day morphine. His most recent pump was replaced in August 2006 from which time he had been maintained on a stable dose of intrathecal hydromorphone $50 \mathrm{mg} / \mathrm{ml}$ at a rate of $17.59 \mathrm{mg} /$ day from 2006 until presenting to our clinic in September 2009. In February 2010, we changed the patient's hydromorphone from $50 \mathrm{mg} / \mathrm{ml}$ to $100 \mathrm{mg} / \mathrm{ml}$ in an effort to reduce refill intervals and maintained the same daily dose of $17.59 \mathrm{mg} /$ day. However, approximately 16 months after increasing the concentration of the intrathecal hydromorphone, the patient developed increasing pain followed by new onset of numbness in his feet. A catheter access study was undertaken which failed to reveal catheter occlusion (Figures $\mathbf{1}$ and 2) and thus the patient was maintained on his daily dose of hydromorphone 17.59 $\mathrm{mg} /$ day at a concentration of $100 \mathrm{mg} / \mathrm{ml}$. Over the next two months the patient continued to complain of increasing pain and was treated with a variety of oral opioid medications. In addition, he began complaining of a gradually progressive ascending numbness from the feet up to his waist and a more pronounced "heaviness" sensation in his legs which had not been present previously. At that point an MRI was obtained (two months after catheter access study) (Figures 3-5). Films were not available for review but concern of a catheter-tip granuloma was raised with the interpreting radiologist. However, the report was read as, "likely [a] hemorrhagic focus abutting the posterior aspect of the spinal cord at T5/6" [with] "a diffusely increased T2 signal intensity

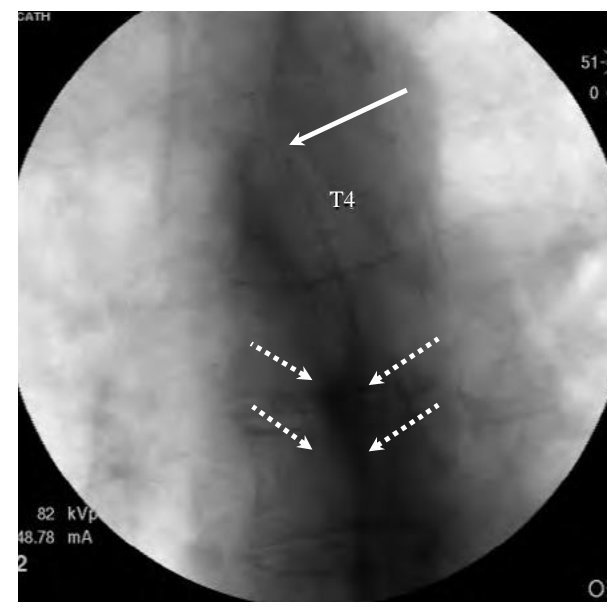

Figure 1. AP fluoroscopic image of catheteraccess study revealing distal end of catheter (solid arrow) and myelogram (dashed arrows).

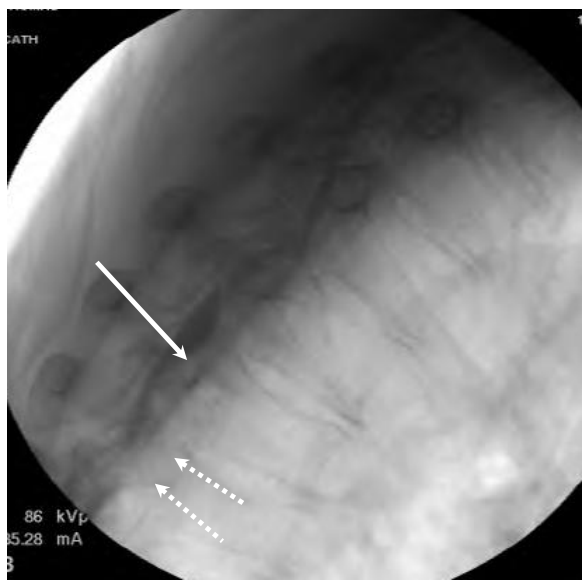

Figure 2. Lateral fluoroscopic view of thoracic spine at catheter end revealing the catheter (solid arrow) and the filling defect (dashed arrows).

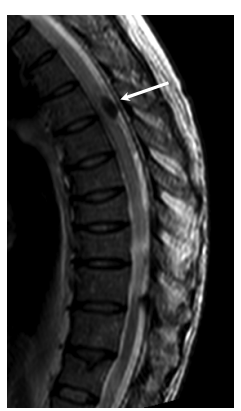

(a)

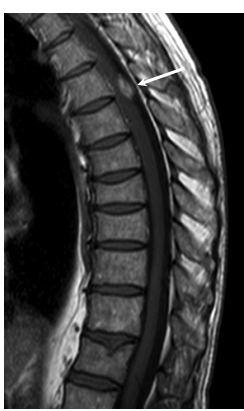

(b)

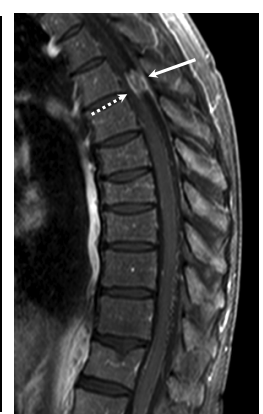

(c)
Figures 3. Sagittal T2-, T1- and postcontrast T1-weighted images demonstrating an extradural intramedullary lesion (solid arrows) with mass effect on the cord. The mass shows low signal on T2-weighted images and high signal on T1-weighted images. There was peripheral enhancement and linear pial enhancement (dashed arrow) with contrast. 


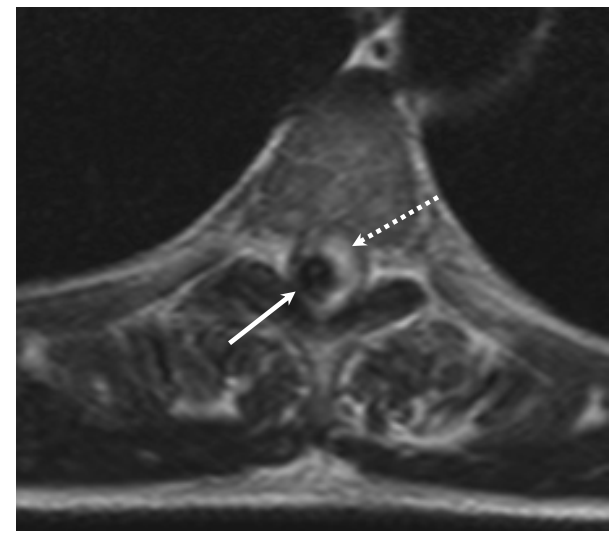

Figure 4. Axial T2-weighted image at the level of the granuloma (solid arrow) shows displacement of the spinal cord anteriorly and to the left (dashed arrow).

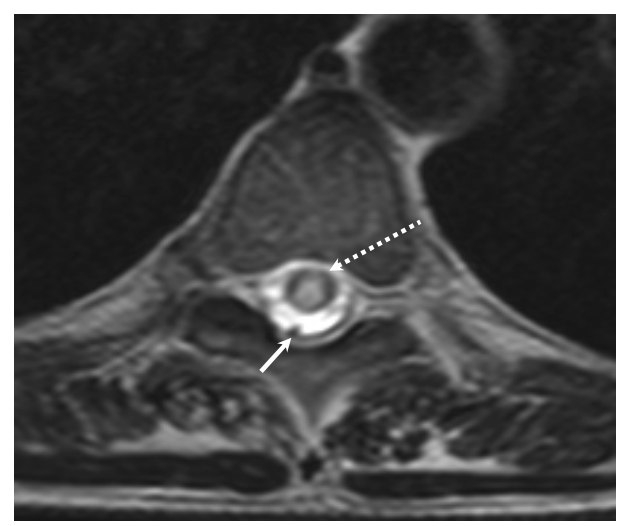

Figure 5. Axial T2-weighted image was obtained caudal to Fig. 4 to demonstrate abnormal high T2-weighted signal in the cord, consistent with edema (dashed arrow). Part of the catheter is seen along the posterior aspect of the canal (solid arrow).

involving the thoracic cord from T2-T9”. Thus, the initial MRI interpretation unfortunately did not appreciate catheter-tip granuloma but rather spinal cord hematoma of uncertain chronicity (Figures 3(a)-(c) and 4). The patient continued to have numbness below the waist with perineal anesthesia and presented to another hospital six days later. On this admission a subsequent MRI revealed a large catheter-tip granuloma which enhanced with contrast, as well as extensive T2 signal prolongation within the spinal cord from T5 - T7 (Figures 6 and 7). The patient underwent semi-urgent surgical exploration, catheter tip removal and excision of the granuloma. However, a portion of the granuloma could not be resected due to tenacious adherence to the spinal cord (Figures 8(a) and (b)). At three months post-operative the patient showed no change in neurologic function with persistent sensory gait ataxia, and hypoesthesia below T6. After approximately five months, repeat MR imaging was obtained revealing recurrent catheter-tip granuloma just inferior to the original granuloma location and, just proximal to the newly trimmed catheter tip (Figures 9(a)-(c)). No changes in the patient's dose or concentration of hydromorphone were made after the first surgery and he continued to receive a daily medication infusion of $17.59 \mathrm{mg} /$ day of hydromorphone $100 \mathrm{mg} / \mathrm{ml}$, in addition to taking Percocet 10/325 mg every 4 - 6 hours orally. Repeat laminectomy and resection of his recurrent granuloma was performed, as well as removal of his catheter and pump system. No significant changes in his clinical condition or neurologic function were observed post-operatively.

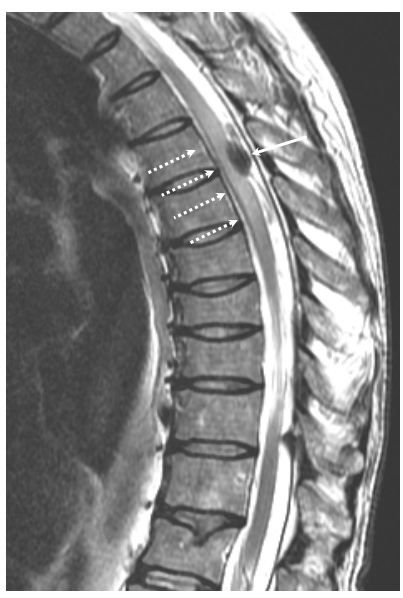

Figure 6. Sagittal T2-weighted image obtained 6 days after inital MRI revealing an intradural lesion (solid arrow) and extensive spinal cord edema (dashed arrows).

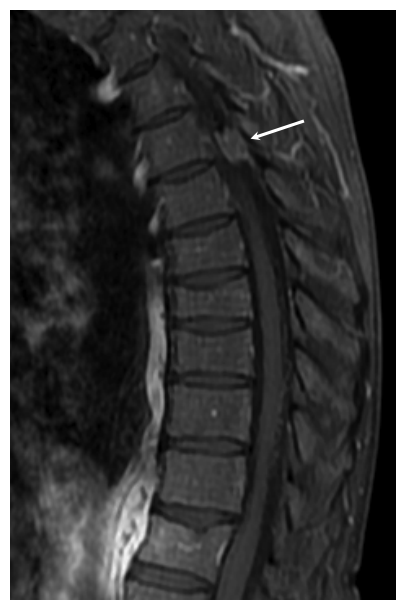

Figure 7. Sagittal T1-weighted fat saturated post-contrast image redemonstrates an enhanceing intramedullary lesion (solid arrow) with peripheral and pial enhancement. 

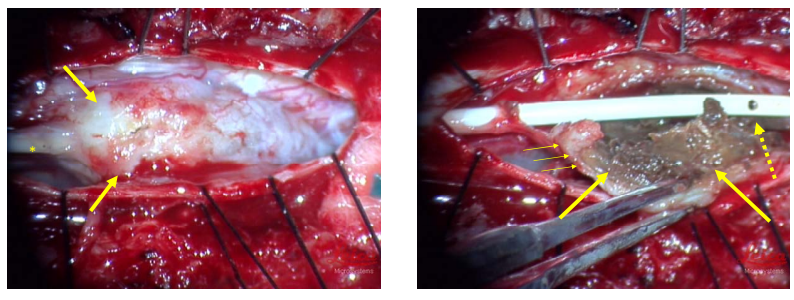

(a)

(b)

Figure 8. (a) Intraoperative image of granuloma (arrow) with dura retracted. Catheter is seen to the left (asterisk); (b) Resection of granuloma from catheter reveals necrotic core of devitalized tissue (arrow) within fibrotic inflammatory layer (small arrows). Note the devitalized mass is proximal to catheter tip near side fenestration (dashed arrow).

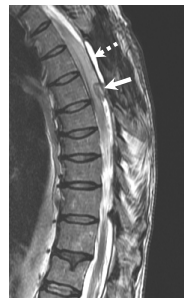

(a)

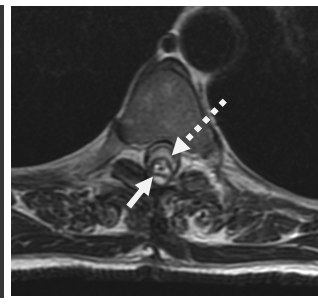

(b)

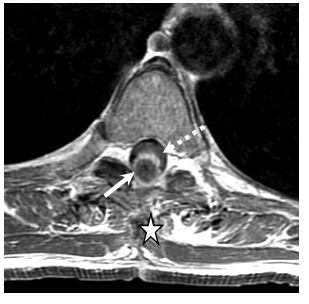

(c)
Figure 9. (a) Sagittal T2-weighted image shows a more caudal location of recurrent catheter-tip granuloma (solid arrow) 4 months after original decompressive surgery. A small seroma is seen cranial and posterior to the recurrent granuloma (dashed arrow); (b) Granuloma (solid arrow) demonstrates high T2weighted signal centrally with peripheral low T2-weighted signal. The spinal cord (dashed arrow) is displaced anteriorly by the mass; (c) Axial T1-weighted post-contrast image reveals peripheral enhancement (solid arrow). Note the post-surgical changes in the posterior elements (star). The spinal cord (dashed arrow) is displaced anteriorly by the mass.

\section{DISCUSSION}

Catheter-tip granulomas remain a rare but potentially devastating complication from intrathecal (IT) infusion therapy [1]. First described after epidural infusion in 1986 [6] and intrathecal infusion in 1991 [3], the number of reported cases has continued to grow with 92 reported cases to the US Food and Drug Administration and Manufacturers as of 2002 [7]. It is likely the true number of cases is much higher given the voluntary reporting basis [8]. Originally used to treat patients with short life expectancies, IT therapy is being increasingly utilized to treat patients with chronic non-malignant pain with approximately 15,000 new pumps implanted each year [1].

Although the majority of cases have been with morphine, hydromorphone may also result in granuloma formation as seen in our case. Thus far, baclofen and diamorphine (heroin) have also been associated with IT granulomas [9]. Preclinical studies have revealed a dosedependent local inflammatory reaction within 28 days at the catheter-tip in Beagle dogs [7] with histopathology of these masses revealing increased vascularity and composition of lymphocytes, macrophages, and ultimately hypervascular fibrous tissue surrounding an inner core of necrotic, devitalized tissue [6,7]. Our case illustrates a concentration-dependent response of granuloma formation to hydromorphone. In addition, our case demonstrates one of the longest time intervals to granuloma diagnosis after implant (approximately 14 years after initial pump implantation and 9 years after catheter revision). The patient had maintained a relatively stable clinical course and was on an extremely steady dose and concentration of hydromorphone for 7 years. However, within 16 months of doubling his hydromorphone concentration, but not changing his daily dose, he developed a clinically significant granuloma. The mass was later resected but then a second granuloma was diagnosed 4.5 months later [10]. Previously, granuloma reformation had been reported to occur as early as 6 to 9 months [11]. Previous case reports have demonstrated recurrence of granulomas up to three times in one individual [9] as well as very fast granuloma formation within 5 weeks of IT morphine therapy [12].

Our case supports the notion that the concentration of medication, rather than the daily dose as originally once thought, was the primary causative agent which precipitated granuloma formation [13]. A 2007 Consensus Conference recommended maximum concentrations of hydromorphone of $10 \mathrm{mg} / \mathrm{ml}$ with maximum daily doses of $4 \mathrm{mg} /$ day [14] intrathecally. However, hydromorphone concentrations as low as $10 \mathrm{mg} / \mathrm{ml}$ and daily doses of 1 $\mathrm{mg}$ /day have been reported to cause granuloma formation [5]. This patient initially was receiving IT morphine $50 \mathrm{mg} / \mathrm{ml}$ combined with clonidine with a daily dose of $32 \mathrm{mg} / \mathrm{ml}$ morphine. Receiving IT clonidine in combination with morphine has been postulated, although not proven, to have a "protective" effect and reduce granuloma formation [7]. However, due to lack of efficacy with no apparent neurologic sequelae this patient was converted to IT hydromorphone. In our case, the hydromorphone concentration reached 10 times the recommended maximum concentration and 4 times the recommended maximum daily dosage of $20 \mathrm{mg} / \mathrm{ml}$ and 4 $\mathrm{mg} /$ day respectively [14].

The location of the catheter-tip has also been suggested to play a role in granuloma formation $[5,7,14]$. Initially this patient had a granuloma form at $\mathrm{T} 5$ and then again at T6 after catheter-tip trimming. Given the relatively small space of cerebrospinal fluid (CSF) at such rostral thoracic locations, some authors have postulated higher catheter-tip placement may predispose to higher local concentrations of drugs at the catheter-tips thus inducing the cellular inflammatory cascade $[5,15]$. Indeed, granulomas that form at catheter-tips placed in- 
ferior to the conus medularis generally result in less severe neurological deficits as well as a more complete recovery should they develop a compressive granuloma [7]. Some IT opioids are lipophilic and thus require placement close to the target spinal segments for maximal effect. We would suggest with hydrophilic medications, such as morphine or hydromorphone which would be expected to spread several segments within the CSF, that catheter-tip location be as caudal as possible in order to secure the catheter and prevent dislodgment.

Although the true yearly incidence of granuloma formation for all pain patients may be dependent on several factors such as the drug, concentration, cathetertip location, daily dose, or patient sensitivity as discussed, the estimated cumulative risk of developing an inflammatory mass was $0.04 \%$ over one year, $0.12 \%$ over two years, but $1.15 \%$ over 6 years [7]. Note the increasing yearly risk such that in this patient who had been implanted approximately 14 years previously, by extrapolating the published data, the estimated risk of granuloma formation would likely be higher than 3\% [7]. More recent publications have placed the incidence of granuloma formation to be as high as $0.49 \%$ to $3 \%[4,16]$. The duration of infusion therapy exposure to granuloma diagnosis ranges from 5 weeks to 72 months, with an average time of 24 months until diagnosis is made [16]. As mentioned, our case represents the longest known duration from initiation of infusion therapy until granuloma diagnosis (14 years).

It is important to note that the majority of radiographically diagnosed granulomas are asymptomatic [16]. Neurologic deficits occurring in response to catheter-tip granulomas are generally slowly progressive and range from loss of drug effect to new-onset of radicular pain or parethesias [5,7,17]. More severe neurological deficits can include paralysis or cauda equina syndrome $[7,2,11]$. Diagnosis in our case was made more difficult in that the patient exhibited opioid seeking behavior with complaints of significant pain and requests for oral analgesics since his initial visit. In addition, the patient had also been discharged from his prior treating physician for recreational drug use. He also had a history of bilateral lower extremity numbness and difficulty with ambulation dating back to his original injury. Our initial evaluation also documented weakness in his lower extremities. As with previous case reports, foot hypoesthesia in this case was the sentinel symptom [11]. The initial catheter access study was undertaken to ensure pump patency and a low index for granuloma formation was entertained given the chronicity and stability of his IT infusion dose and medication. As previously mentioned, this study was originally interpreted as normal, however, on further review of his catheter access study myelogram (Figure 2) a filling defect located just proximal to the catheter-tip can be appreciated, which is consistent with a side fenestrated catheter. Our patient remained ambulatory both before and after each granuloma excision with minimal clinical improvement.

Catheter-tip granulomas, although well reported in the neurosurgical and pain medicine literature are less well described in the radiological literature [3]. In this particular case, diagnosis of the granuloma was delayed slightly as it was initially interpreted as a "hemorrhagic focus” abutting the spinal cord. (Figures 3(a)-(c)). Relaying pertinent clinical information to interpreting radiologist may aid in diagnosis and hasten treatment. In addition, documenting catheter-tip location as well as model type (end hole vs. side fenestration) in the patient's medical record at the time of implant may facilitate diagnosis [17]. The patient's first MRI revealed the typical ring-like enhancement on contrast-enhanced T1-weighted imaging (Figure 3(c)). Because some catheters have a metallic marker at the tip, contrast-enhancement is crucial [3]. As seen in Figure 8(b), the peripheral fibrotic, hypervascular shell surrounding the inner necrotic core explains the peripheral ring-like enhancement.

Treatment of catheter-tip masses should take into account the patient's clinical condition, as well as the physician's experience [17]. Once a catheter-tip mass is radiographically confirmed, a decision to leave the drug infusion system in place or remove all or part of it must be made. If the decision to leave the infusion system in place is made the clinician must decide whether to change the dose, concentration, or medication administered altogether [17]. Some clinicians may also choose to pull the catheter back one or two caudal segments under light sedation [16]. Although multiple exit catheters may have an advantage over single exit catheters [16], this patient in fact had a six-orificed, side-fenestrated catheter. If a compressive lesion is suspected and surgery contemplated, the pump should be stopped immediately and the patient should be referred to a spine surgeon. However, stopping the pump for more than a few days may damage the catheter near the rotors [16]. In our case the patient underwent semi-urgent surgical granuloma excision and catheter trimming without any changes to the concentration and daily dose of his medication. We, however, recommend medication adjustments be made in future similar situations.

In summary, benefits of IT therapy include localized and improved analgesia, decreased side effects compared with systemic medications and more alert, less hypersomnolent patients when compared to oral opioids [18]. Although rare, catheter-tip granulomas remain a serious complication of IT infusion therapy and clinicians managing patients with implanted subarachnoid infusion pumps should maintain a high index of suspicion for granuloma formation in any patient with decreasing res- 
ponsiveness to therapy or subtle changes in neurologic function. We believe this case represents the longest interval to diagnosis after initiation of IT infusion therapy, as well as one of the shortest intervals of recurrence of a catheter-tip mass. In addition, the filling defect during the catheter access study was not appreciated, nor was the granuloma appreciated on the patient's initial MRI. This case report is intended to educate practitioners in recognizing the presentation of a possible catheter-tip granuloma, both clinically and radiographically, to help improve recognition and institute treatment in a quick and effective manner, and to decrease the prevalence of mistakenly unidentified catheter-tip granulomas.

\section{ACKNOWLEDGEMENTS}

The authors would like to gratefully acknowledge Ashley Zimmerman, PA-C for her editorial assistance in preparation of this manuscript.

\section{REFERENCES}

[1] Medtronic Internal Communication (2010) UC201003915 EN NP9832 C, Medtronic, Inc.

[2] North, R.B., Cutchis, P.N., Epstein, J.A. and Long, D.M. (1991) Spinal cord compression complicating subarachnoid infusion of morphine: Case report and laboratory experience. Neurosurgery, 29, 778-784. doi:10.1227/00006123-199111000-00025

[3] Phillips, J.A., Escott, E.J., Moosey, J.J. and Kellermier, H.C. (2007) Imaging appearance of intrathecal catheter tip granulomas: Report of three cases and review of the literature. American Journal of Roentgenology, 189, W375W381. doi:10.2214/AJR.07.2225

[4] Ramsey, C.N., Owen, R.D., Witt, W.O. and Grider, J.S. (2008) Intrathecal granuloma in a patient receiving high dose hydromorphone. Pain Physician, 11, 369-373.

[5] Coffey, R.J. and Burchiel, K.B. (2002) Inflammatory mass lesions associated with intrathecal drug infusion catheters: Report and observations on 41 patients. Neurosurgery, 50, 78-86.

[6] Rodan, B.A., Cohen, F.L., Bean, W.J. and Martyak, S.N. (1985) Fibrous mass complicating epidural morphine infusion. Neurosurgery, 16, 68-70. doi:10.1227/00006123-198501000-00014

[7] Yaksh, T.L., Hassenbusch, S., Burchiel, K., Hildebrand, K.R., Page, L.M. and Coffey, R.J. (2002) Inflammatory masses associated with intrathecal drug infusion: A review of preclinical evidence and human data. Pain Medicine, 3, 300-312. doi:10.1046/j.1526-4637.2002.02048.x

[8] Follett, K.A. (2003) Intrathecal analgesia and catheter-tip inflammatory masses. Anesthesiology, 99, 5-6. doi:10.1097/00000542-200307000-00004
[9] Miele, V.J., Price, K.O., Bloomfield, S., Hogg, J. and Bailes, J.E. (2006) A review of intrathecal morphine therapy related granulomas. European Journal of Pain, 10, 251261. doi:10.1016/j.ejpain.2005.05.002

[10] Andrés, J.D., Palmisani, S., Villanueva Pérez, V.L., Ansensio, J. and Lopez-Alcaron, M.D. (2010) Can an intrathecal, catheter-tip-associated inflammatory mass reoccur? The Clinical Journal of Pain, 26, 631-364. doi:10.1097/AJP.0b013e3181e4a541

[11] Hoederath, P., Gautschi, O.P., Land, M., Hildebrandt, G. and Fournier, J.Y. (2010) Formation of two consecutive intrathecal catheter tip granulomas within nine months. Central European Neurosurgery, 71, 39-42. doi:10.1055/s-0029-1202359

[12] Jourdain, V., Cantin, L., Prud'Homme, M. and FournierGosselin, M.P. (2009) Intrathecal morphine therapy-related granuloma: Faster to grow then thought. Neuromodulation, 12, 164-168. doi:10.1111/j.1525-1403.2009.00205.x

[13] Cabbell, K.L., Taren, J.A. and Sager, O. (1998) Spinal cord compression by catheter granulomas in high-dose intrathecal morphine therapy: Case report. Neurosurgery, 42, 1176-1181. doi:10.1097/00006123-199805000-00142

[14] Deer, T., Krames, E.S., Hassenbusch, S.J., Burton, A., Caraway, D., Dupen, S., Eisenach, J., Erdek, M., Grigsby, E., Kim, P., Levy, R., McDowell, G., Mekhail, N., Panchal, S., Prager, J., Rauck, R., Saulino, M., Sitzman, T., Staats, P., Stanton-Hicks, M., Stearns, L., Willis, K.D., Witt, W., Follett, K., Huntoon, M., Liem, L., Rathmell, J., Wallace, M., Buchser, E., Cousins, M. and Donck, A.V. (2007) Polyanalgesic consensus conference 2007: Recommendations for the management of pain by intrathecal (intraspinal) drug delivery: Report of an interdisciplinary expert panel. Neuromodulation, 10, 300-328. doi:10.1111/j.1525-1403.2007.00128.x

[15] Medel, R., Pouratian, N. and Elias, W.J. (2010) Cathetertip mass mimicking a spinal epidural hematoma. Neurosurgery, 12, 66-71.

[16] Deer, T.R. (2004) A prospective analysis of intrathecal granuloma in chronic pain patients: A review of the literature and report of a surveillance study. Pain Physician, 7, 225-228.

[17] Hassenbusch, S., Burchiel, K., Coffey, R.J., Cousins, M.J., Deer, T., Hahn, M., Du Pen, S., Follett, K.A., Krames, E., Rogers, J.N., Sagher, O., Staats, P.S., Wallace, M. and Willis, K.D. (2002) Management of intrathecal cathetertip inflammatory masses: A consensus statement. Pain Medicine, 3, 313-323. doi:10.1046/j.1526-4637.2002.02055.X

[18] Blount, J.P., Remley, K.B., Yue, S.K. and Erickson, D.L. (1986) Intrathecal granuloma complicating chronic spinal infusion of morphine. Journal of Neurosurgery, 84, 272276. doi:10.3171/jns.1996.84.2.0272 